\title{
FPGA Implementation of LTE Downlink Transceiver with Synchronization and Equalization
}

\author{
Sara M. Hassan \\ Modern Academy, \\ Cairo, Egypt
}

\author{
Abdelhalim Zekry \\ Ain Shams University, \\ Cairo, Egypt
}

\begin{abstract}
Long Term Evolution (LTE) is an advanced standard of the mobile communication systems. LTE has been developed by the $3^{\text {rd }}$ Generation Partnership Project (3GPP). The new features exhibited by LTE is a direct impact of applying new modulation and coding techniques such as the Orthogonal Frequency Division Multiplexing (OFDM) for the Downlink and the Single Carrier Frequency Division Multiple Access (SC-FDMA) for the Uplink as well as turbo coding. This paper presents a Field Programmable Gate Array (FPGA) design and implementation of the LTE downlink transmitter and receiver according to releases 8 and 9 on Virtex 6 XC6VLX240T FPGA kit using Xilinx® ISE® Design Suite version 12.1. It is found that the utilization of the look up tables and flip plops amounts to about 65 percent while the other logic devices utilization on the chip amount to only 513 percent. Such implementations can be considered as IPs for software defined radios. The information is also useful for the FPGA developers. The most important consequence is that the FPGA vendors may produce more appropriate counts of the resource blocks for better the utilization of the chips used in the LTE transceivers.
\end{abstract}

\section{Keywords}

LTE, 4G, 3GPP, OFDM, 2G ,3G, LTE downlink physical layer, release 8, release 9 Xilinx Design Suite, virtex 6 XC6VLX240T FPGA.

\section{INTRODUCTION}

The Long Term Evolution (LTE) is an advanced standard for wireless voice and data communication., The 3GPP standards' body has completed definition of the first release of the LTE system, The main advantages of the LTE, also known as 4G, over the $2 \mathrm{G}$ and $3 \mathrm{G}$ systems are utilizing a higher peak data rates by providing for an uplink speed of up to 50 megabits per second (Mbps) and a downlink speed of up to $100 \mathrm{Mbps}$. LTE will bring many technical benefits to cellular NETWORKS. BANDWIDTH WILL BE SCALABLE FROM 1.25 MHz TO $20 \mathrm{MHz}$. This will suit the needs of different network operators that have different bandwidth allocations, and also allow operators to provide different services based on spectrum. LTE also improved spectral efficiency up to $5 \mathrm{bps} / \mathrm{Hz}$ for Downlink and $2.5 \mathrm{bps} / \mathrm{Hz}$ for Uplink, allowing carriers to provide more data and voice services over a given bandwidth.

The LTE physical layer (PHY) is a highly efficient means of conveying both data and control information between an enhanced base station (eNodeB) and mobile user equipment (UE). The LTE PHY layer employs some advanced technologies that are new to cellular applications. These include Orthogonal Frequency Division Multiplexing (OFDM) and Multiple Input Multiple Output (MIMO) data transmission. In addition, the LTE PHY layer uses Orthogonal
Frequency Division Multiple Access (OFDMA) on the downlink (DL) and Single Carrier - Frequency Division Multiple Access (SC-FDMA) on the uplink (UL). OFDMA allows data to be directed to or from multiple users on a subcarrier-by-subcarrier basis for a specified number of symbol periods. Due to the novelty of these technologies in cellular applications, they are described separately before delving into a description of the LTE PHY layer.

LTE uplink requirements differ from downlink requirements in several ways. Not surprisingly, power consumption is a key consideration for UE terminals. The high PAPR and related loss of efficiency associated with OFDM signaling are major concerns. As a result, an alternative to OFDM was sought for use in the LTE uplink.

Single Carrier - Frequency Domain Multiple Access (SCFDMA) is well suited to the LTE uplink requirements. The basic transmitter and receiver architecture is very similar (nearly identical) to OFDMA, and it offers the same degree of multipath protection. Importantly, because the underlying waveform is essentially single-carrier, the PAPR is lower [15].

The continued increase in the number of users of mobile communications all over the world, motivated researchers to search for a unified wireless platform. This will enable the mobile users to conduct business and exchange data easily while moving elsewhere in the world. The new features of LTE made it the promising platform intended for the advancements in mobile communications [6].

In the last few years, technology evolution in mobile communications is mainly motivated by two relevant agents: (1) the market globalization and liberalization and the increasing competence among vendors and operators coming from this new framework, (2) the exponential increase in the demand for advanced telecommunication services and we note that the computational framework and the insights gained via the numerical studies can be extended to other orthogonal division frequency multiple access (OFDMA) technologies, For that the many researchers are concerned with the development of implementation methods and techniques for the building blocks of the LTE physical layer. So, an extensive research work is directed to the implementation of the building blocks of this system using different platforms [7-10].

This paper presents the simulation and the FPGA implementation of the LTE downlink physical layer (on Virtex 6 XC6VLX240T FPGA kit) according to release 9 using Xilinx package version 12.1. Every stage in both the transmitter and receiver are implemented, and verified. The paper is organized such that section 2 presents the full detailed description of the building blocks of the LTE transmitter and 
receiver. Section 3 introduces the design verification while section four gives data about the system utilization of the Xilinix Vertix 6 and section fie concludes the paper.

\section{TRANSIMITTER AND RECIVER IMPLEMENTATION}

The LTE implementation in this paper is built according to specifications of LTE in release 9 with FDD (Frequency Division Duplex) frame structure. The implemented system has a carrier bandwidth of $3 \mathrm{GHz}$, the number of sub-channels is 15 , the number of sub-carrier is 180 , the IFFT/FFT size is 256 , the turbo encoder/decoder rate is $1 / 3$, and the data input for one OFDM symbol has a length of 96 bits [11].

Figure 1 and Figure 2 show the implemented transmitter and receiver block diagrams, respectively. All the operations done in the transmitter have to be done reversely in the receiver. The reverse operation of the cyclic redundancy check (CRC) in the transmitter is the DE-CRC in the receiver, the segmentation block in the transmitter corresponds to DESegmentation in the receiver, the turbo encoder in the transmitter corresponds to Decoder in the receiver, the rate matching block in the transmitter corresponds to De- rate matching in the receiver, the scrambler in the transmitter corresponds to De-Scrambler in the receiver, the mapper in the transmitter corresponds to De-mapper in the receiver, the inverse Fourier transform block (IFFT) in the transmitter corresponds to FFT in the receiver, and the cyclic prefix insertion block in the transmitter corresponds to cyclic prefix remove in the receiver.

In the following sections, we will show the VHDL model and FPGA implementation of the basic building blocks of the transmitter and the corresponding blocks in the receiver. Moreover, the verification of each block and its reverse operation will be shown. Finally overall system (transmitter/receiver) verification will be done.

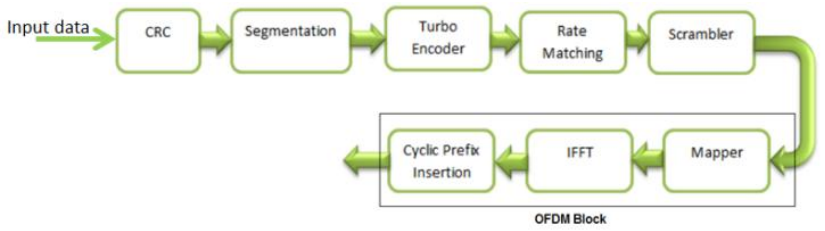

Fig 1: The blocEk diagram of physical layer of the LTE downlink transmitter [11]

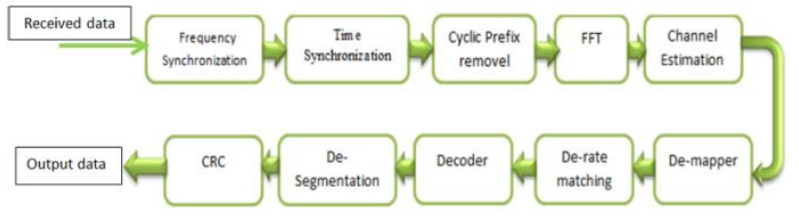

Fig 2: The receiver block diagram [14]

\subsection{CRC and DE-CRC implementation}

The first block in the transmitter is the CRC; which is an independent block. The function of this block is to add 24 redundancy bits at the start of each transport block for sake of detecting errors in the whole transport block. According to the specifications, the polynomial used in this block is called " $\mathrm{g}_{\text {CRC24A }}$ " and it follows the equation [12]:

$\mathrm{g}_{\text {CRC24A }}(\mathrm{D})=\left[\mathrm{D}^{24}+\mathrm{D}^{23}+\mathrm{D}^{18}+\mathrm{D}^{17}+\mathrm{D}^{14}+\mathrm{D}^{11}+\mathrm{D}^{10}+\mathrm{D}^{7}+\right.$ $\left.\mathrm{D}^{6}+\mathrm{D}^{5}+\mathrm{D}^{4}+\mathrm{D}^{3}+\mathrm{D}+1\right]$.

Where Dn is the location that will be occupied by "1".
The transport block data is modulo 2 divided by this generator polynomial of 24 bits sequence and the remainder is the CRC bit sequence that will be added to the start of the transport block. This block is modeled using the hardware description language VHDL and implemented on Xilinix Vertix 6 FPGA chip. The implementation is validated by assuming an input data sequence of 25 ones, then the CRC output data must be '011110011011001100000100'. Figure 3 shows the results of the implemented CRC block. It is shown that the number of input bits to the CRC block is 25 bits. The number of the CRC bits is 24 bits. Consequently the number of the output bits is 49 (input + CRC bits)

The DE-CRC process is implemented in the receiver. This process is executed in the same way as in the transmitter where the received data bits ( 25 bits) are used to generate 24 CRC bits following the same processing steps that made at the transmitter. Figure 4 shows the simulation of the DE-CRC block. The error indicator output flag is zero which means the CRC-bits regenerated at the receiver is the same as the CRCbits generated at the transmitter. This verifies the CRC and DE-CRC blocks.

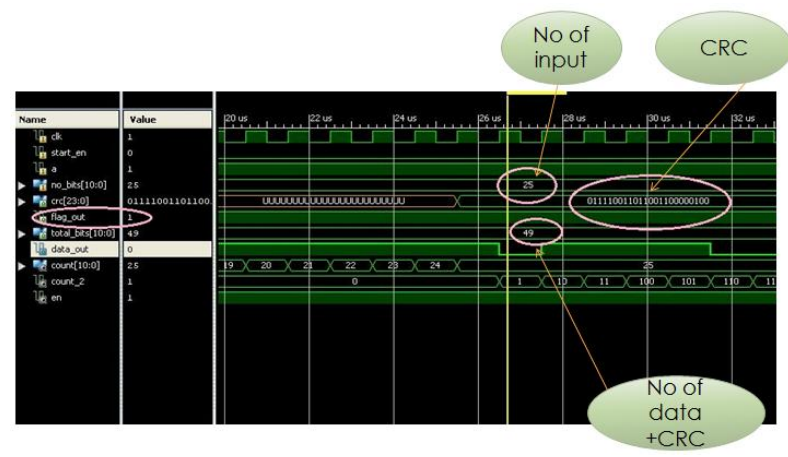

Fig 3: The simulation results of the CRC block

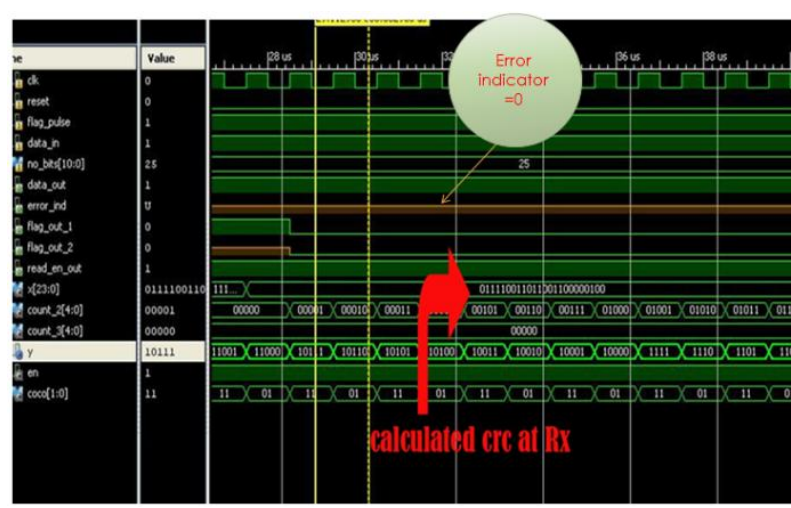

Fig 4: The simulation results of the DE-CRC block

\subsection{SEGMENTATION and DE- SEGMENTATION}

The Turbo Encoder can handle the data correctly if only its input transport block size is less than 6144. In case the length of the input transport block is greater than 6144, then the transport block is split into multiple segments. In this case, a CRC code is added to every segment to detect the error in it. The CRC inside the segmented block is calculated according to a different polynomial than that used previously in the first CRC block. The CRC generating polynomial used is called" $\mathrm{g}_{\mathrm{CRC} 24 \mathrm{~B}}$ " and is expressed by the following equation [12]: 
$\mathrm{g}_{\mathrm{CRC} 24 \mathrm{~B}}(\mathrm{D})=\left[\mathrm{D}^{24}+\mathrm{D}^{23}+\mathrm{D}^{6}+\mathrm{D}^{5}+\mathrm{D}+1\right]$.

The output of the first CRC block, which comprises 49 bits, is utilized as an input to the segmentation block. The 49 bits are segmented into 3 equal segments. The segmentation process is executed according to the standards of release 9 section (5.1.2) and the length of each segment is determined according to table (5.1.3-3) [12]. The simulation results of the segmentation building block are shown in Figure 5.

The DE-segmentation process is the reverse operation of the segmentation process, that by reassemble the received segments at the receiver. The DE segmentation building block is designed and implemented by a VHDL code and it function is verified in the same manner as the segmentation block.
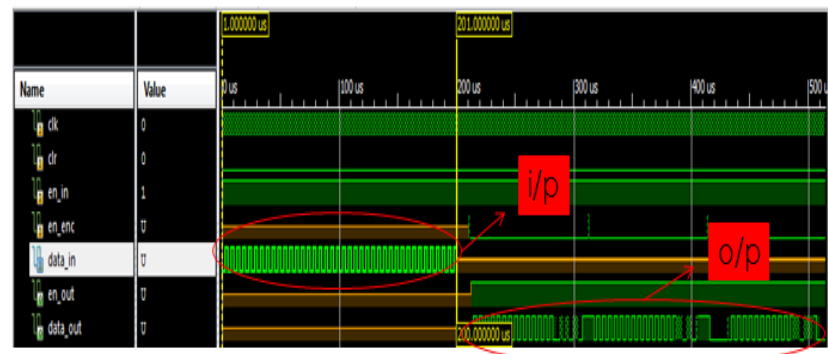

Fig 5: The testing results of the segmentation block.

\subsection{TURBO ENCODER AND DECODER}

In the following subsections, we will show the Turbo Encoder and Decoder implementation and its verification.

\subsubsection{Turbo Encoder}

The third block in the LTE transmitter is the Turbo Encoder. The Parallel Concatenated Convolutional Code (PCCC) is the scheme used in the Turbo Encoder. As shown in Figure 6, this scheme consists of two 8-state encoders and one turbo code internal interleaver. The coding rate of this turbo encoder is 1/3. The input to the Turbo Encoder is the output of the Segmentation block; with a length of 100 bits. The three outputs of the Encoder are the systematic sequence $d_{K}^{(0)}$ and two sequences of parity bits $d_{K}^{(1)}$, and $d_{K}^{(2)}$. The length of each one of the three outputs is 100 bits. The systematic sequence ( $\left.d_{K}^{(0)}\right)$ is the same as the input sequence Turbo encoder $c_{K}$, while the two parity sequences of the two parity sequences ( $\left.d_{K}^{(1)}, d_{K}^{(2)}\right)$ are calculated according to the logic circuit shown in Figure 6. In turbo encoder the output sequences of length $\mathrm{K}$ must be terminated by padding 3 consequent zeros to the input sequence.

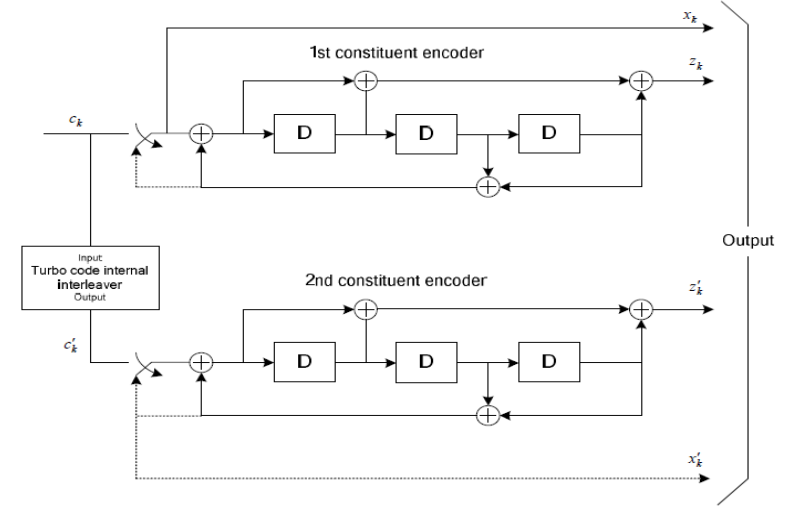

Fig 6: Structure of rate 1/3 turbo encoder [11]
Then, the transmitted bits for trellis termination shall then be [11]:

$$
\begin{aligned}
& d_{K}^{(0)}=x_{K}, d_{K+1}^{(0)}=z_{K+1}, d_{K+2}^{(0)}=x_{K}^{\prime}, d_{K+3}^{(0)}=z_{K+1}^{\prime} \\
& d_{K}^{(1)}=z_{K}, d_{K+1}^{(1)}=x_{K+2}, d_{K+2}^{(1)}=z_{K}^{\prime}, d_{K+3}^{(1)}=x_{K+2}^{\prime} \\
& d_{K}^{(2)}=x_{K+1}, d_{K+1}^{(2)}=z_{K+2}, d_{K+2}^{(2)}=x_{K+1}^{\prime}, \\
& d_{K+3}^{(2)}=z_{K+2}^{\prime},
\end{aligned}
$$

Where $K$ is the number of input bits.

The turbo encoder is implemented by a Xilinx core. The results of the implemented turbo encoder generated by Xilinix core is shown in Figure 7. The input data sequence and the three outputs (schematic, parity1, and parity2) are clearly seen in the figure.

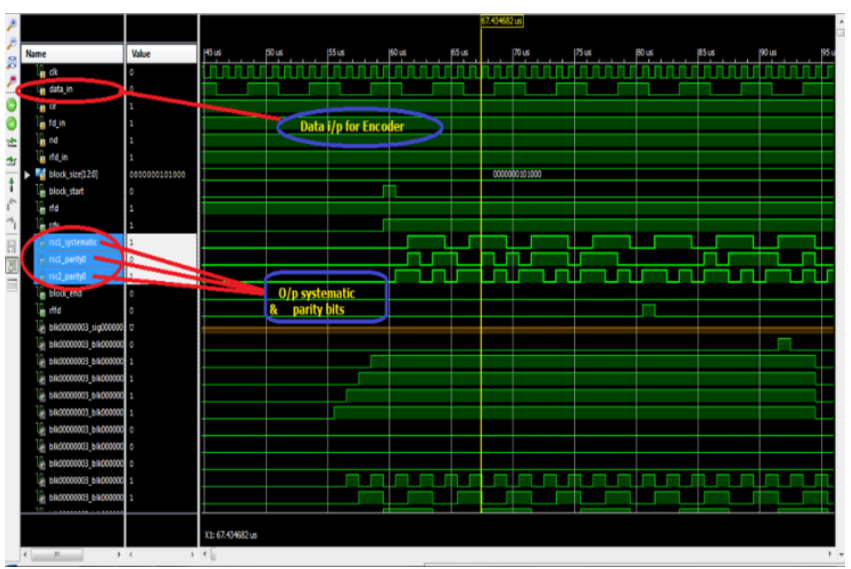

Fig 7: The implementation results of the turbo encoder.

\subsubsection{The Turbo Decoder}

The Turbo Decoder process is the reverse operation of the turbo encoder. The Decoder is implemented by a Xilinx core. Figure 8 shows the testing results of the implemented decoder. The three inputs (schematic, parity1, and parity2) and output data sequences are clearly seen in the Figure.

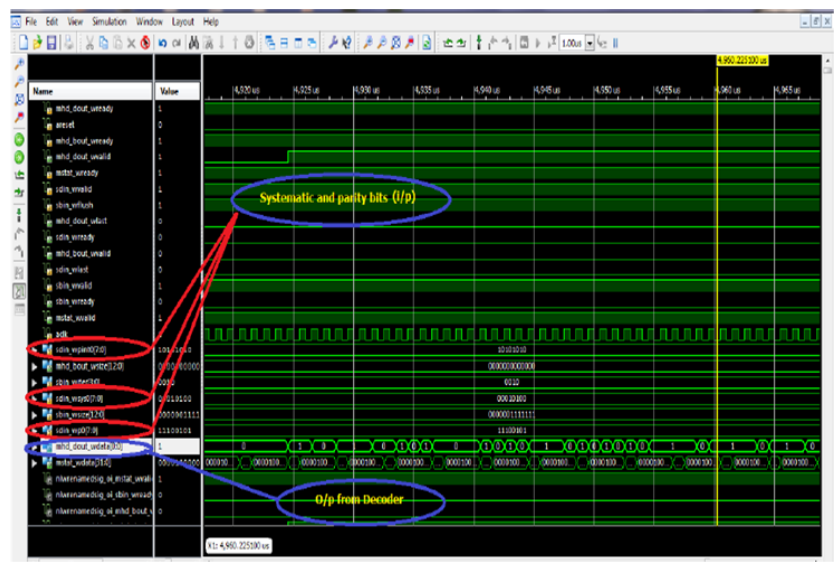

Fig 8: The simulation results of the decoder

\subsubsection{Turbo Encoder and Decoder verification}

The verification is accomplished by the Loop back test where the output of the decoder is input to the decoder. The Turbo Encoder and the Decoder verification is passed when the output of the Decoder is identical to the input of the Turbo Encoder. The verification test results of the Encoder and 
Decoder is depicted in Figure 9. It is clear from the figure that the data output from the Decoder is the same as the data input to the Encoder.

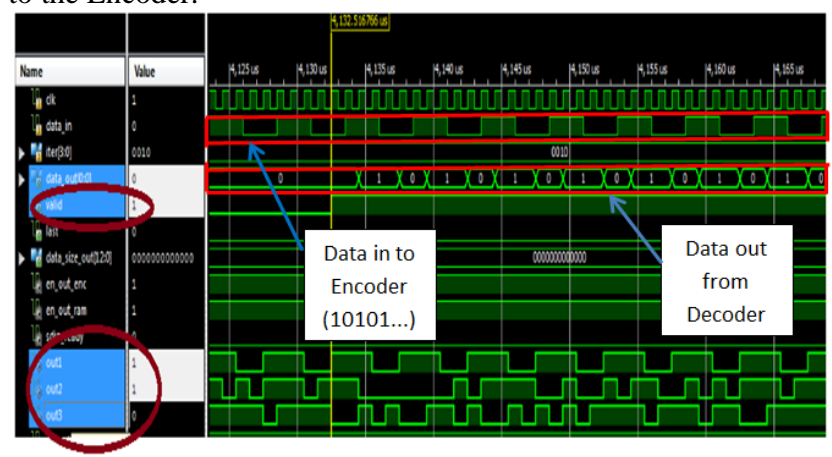

Fig 9: The encoder and decoder verification

\subsection{RATE MATCHING AND DE-RATE MATCHING}

In the following subsections, we will introduce the Rate matching and De-rate matching implementation and functional verification.

\subsubsection{Rate Mathing}

The next stage to the turbo encoder is the Rate Matching block. The function of this block is to add more security and immunity for the transmitted data against the channel errors. Figure 10 shows the schematic diagram of the rate matching block.

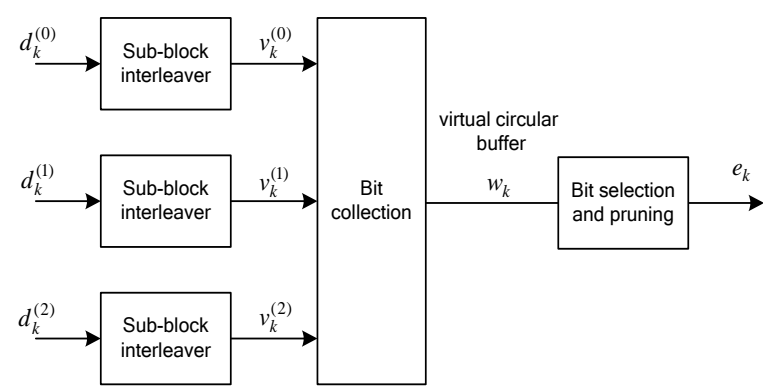

Fig 10: Rate matching block for turbo coded transport channels [12]

The operation of the Sub-block interleaver is to reorder the positions of the input bit stream according to Table 1 .

Table 1. Inter-column permutation pattern for sub-block interleaver [12]

\begin{tabular}{|c|c|}
\hline $\begin{array}{c}\text { Number of columns } \\
C_{\text {subblock }}^{c C}\end{array}$ & $\begin{array}{c}\text { Inter.column permutation pattem } \\
\left\langle P(0), P(1), \ldots, P\left(C_{\text {subblock }}^{c C}-1\right)\right\rangle\end{array}$ \\
\hline \hline 32 & $\langle 1,17,9,25,5,21,13,29,3,19,11,27,7,23,15,31$, \\
\hline \hline
\end{tabular}

The function of the bit collection is to group the three sequences into one output sequence by taking all the bits of the $v_{k}(0)$ followed by a bit alternation between $v_{k}(1)$ and $v_{k}(2)$. The bit collection operation is shown in Figure 11.

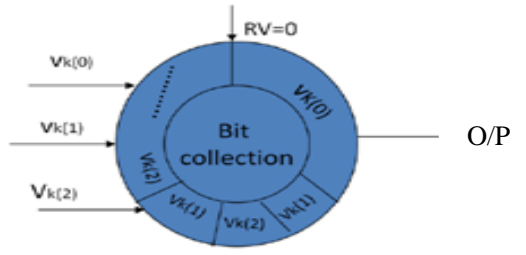

Fig 11: The transmission bit collection and selection operation

Figure $12 \mathrm{a}$ and $12 \mathrm{~b}$ show the simulation results of the implemented Rate Matching block. Figure 12a depicts the three input streams before and after the interleaving process while Figure $12 \mathrm{~b}$ indicates the output stream of the Rate Matching block after combining the three interleaved streams.

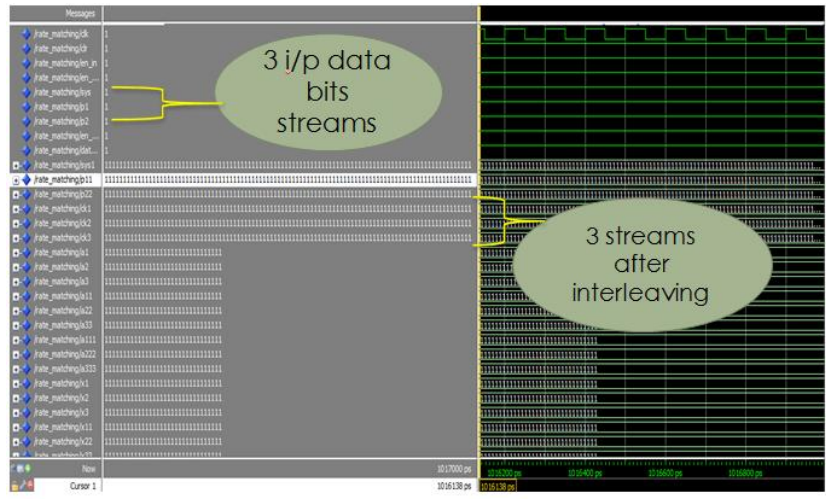

Fig 12a: Simulation results of the interleaving in the Rate matching operations

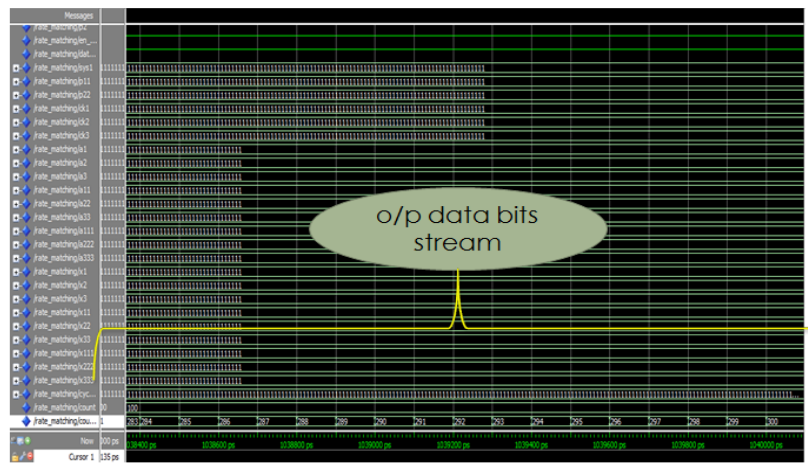

Fig 12b: Simulation results of the stream combining in Rate matching operation

\subsubsection{DE-Rate Matching}

The DE-rate matching processes are the reverse operations of rate matching, that by applying the received data into bit recollection block to generate the original order sequences of three sub blocks as they were ordered in the transmitter. Then every sub block is de-interleaved to restore the original data order as they were at the input of the rate matching block. This building block is implemented and Figure 13 shows its simulation results. It is clear from the figure that the input data results in three output streams representing the restored original data. 


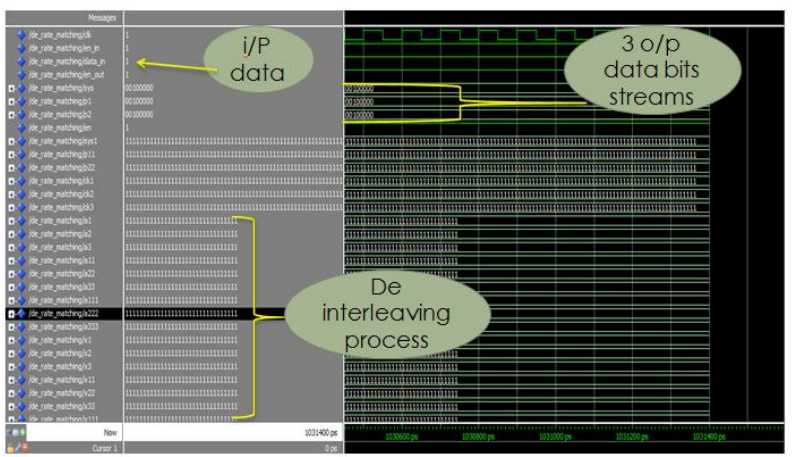

Fig 13: Simulation results of DE-Rate matching operations

\subsubsection{Rate Matching and DE-Rate Matching verification}

The functionality of the Rate Matching and the De-rate matching blocks are verified by the loop back test where the output data of the Rate Matching block is directly connected as an input to the De-rate matching block. Then if they function correctly the output the De-rate matching must be identical to the input of the Rate Matching. This test is executed and the results are illustrated in Figure 14.

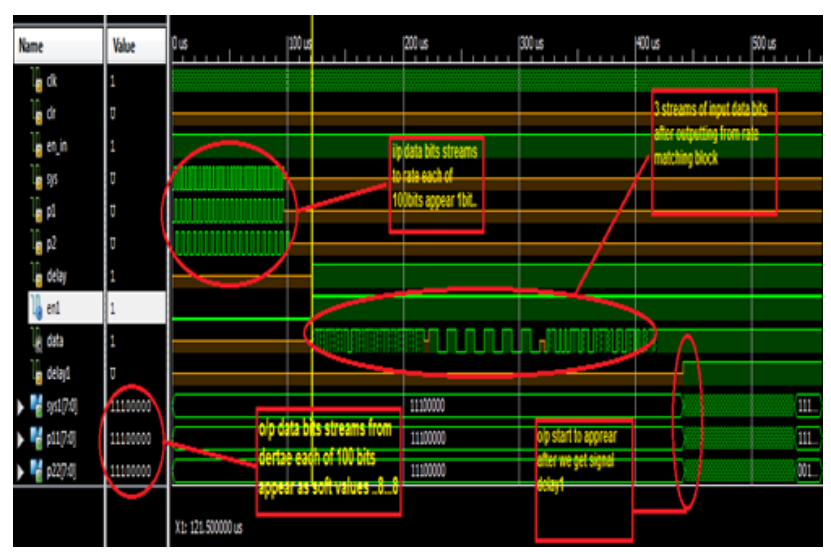

Fig 14: The Rate Matching and De-rate Matching verification

By inspecting the figure it is clear that the three inputs to the Rate matching block at the transmitter; which are systematic, parity 1 , and parity 2 streams, are the same as the output of the DE Rate matching block at the receiver.

\subsection{THE SCRAMBLER AND DE- SCRAMBLER}

\subsubsection{The Scrambler}

The next stage after turbo encoder is the Scrambler. There are two main functions for the Scrambler; increasing the system security and preventing long sequences of ones or zeros in the transmitted data to facilitate the clock regeneration in the receiver. The two functions are done through using a Pseudo Random Sequence Generator (PRSG) having a length of 31 bits that is Xored with the data to produce the Scrambler output.

Following the same procedure as with the previous building blocks, The test results of the implemented scrambler is shown in Figure 15, where "data_in" is the input data to the Scrambler, "q_out" is the output from the PRSG, and "data_out" is the output data from Scrambler such that (data_out $=$ data_in Xor q_out).

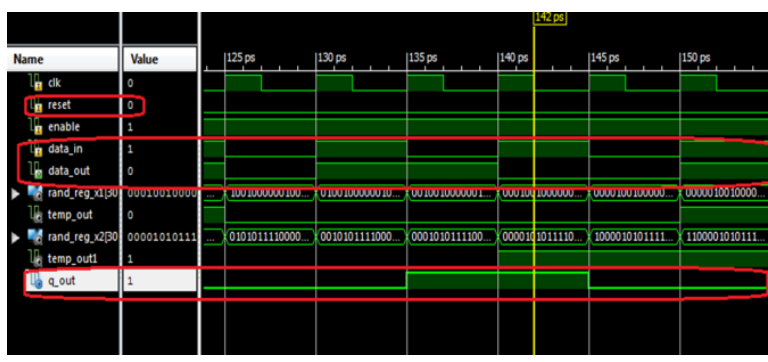

Fig 15: The simulation results of the scrambler

\subsubsection{The DE-Scrambler}

The DE-scrambler process is the reverse of the scrambler process that by Xoring the received data with the " $q$ out" sequence, the same one at the transmitter, restores the original data as that input to the scrambler produces the De-scrambler output. The test results of the implemented DE-scrambler is shown in Figure 16, where "data_in" is the received input data to DE-Scrambler, "q_out" is the output from the PRSG, and "data_out" is the output data from DE-Scrambler.

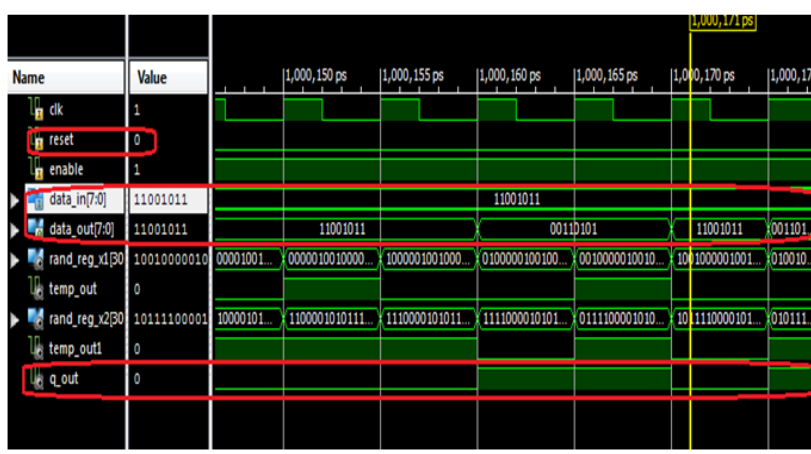

Fig 16: The simulation results of the DE-scrambler

\subsubsection{Scrambler and DE- Scrambler verification}

The Scrambler and the Descrambler verification is carried out by the conventional loop back test where the output of the descrambler is directly input to the descrambler. If the output of the descrambler is identical to the input of scrambler, then the design is correct. Figure 17 depicts the results of the loop back test where one sees clearly that the output the Descrambler is identical to the input of the Scrambler. The Scrambler and Descrambler verification is shown in figure 17.

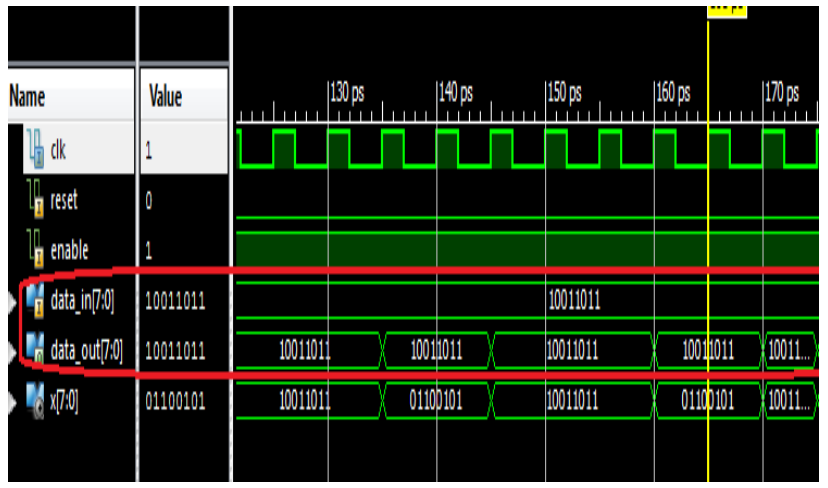

Fig 17: The scrambler and de scrambler verification

It is shown that the output from the DE scrambler is the same as the input to the Scrambler. 


\subsection{THE OFDM BLOCK}

The next stage after the scrambler is the orthogonal frequency division multiplexer, OFDM block. The OFDM block diagram is shown in figure 18. It consists of a mapper, a pilot generator, a data and pilot insertion block and an IFFT core.

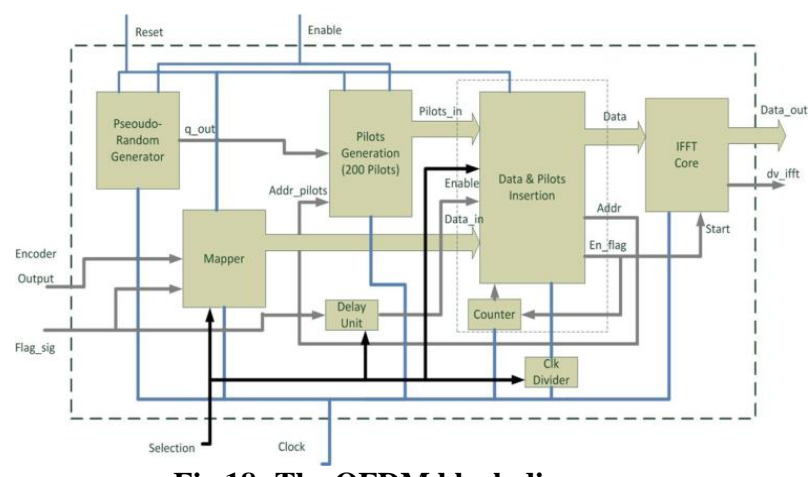

Fig 18: The OFDM block diagram

The Pseudo Random Sequence Generator (PRSG) is built as the same in the scrambler block. The pilot generator produces pilot values and save them in a RAM of length 200 words and width of 22 bits. These pilots are used at the receiver to estimate the channel transfer characteristics and hence make channel equalization. Figure 19 shows the generated pilots.

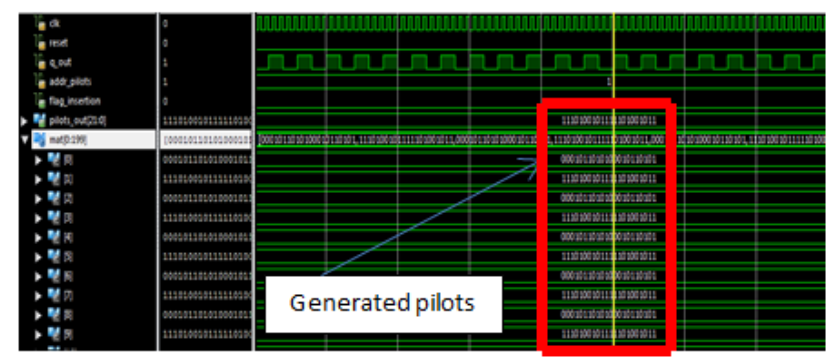

Fig 19: The generated pilots

Both the output data of the Mapper block and the output data of the Pilot Generator do not arrive at the same time at the Data and Pilot Insertion Random access memory, RAM. So, the Delay unit was implemented to solve this problem. Now we introduce the major sub blocks of the OFDM block.

\subsubsection{Mapper}

The mapper realizes the constellation diagram of the digital modulation of the type M-QAM with $\mathrm{M}=2,4,16$, and 64, where certain logic number is assigned to a baseband symbol having specific I and Q components, with I and Q the in-phase and quadrature component, respectively. The RTL Schematic of the mapper with $\mathrm{M}=4$ is shown in Figure 20 while the testing results and signals of the QAM mapper are indicated in Figure 21.

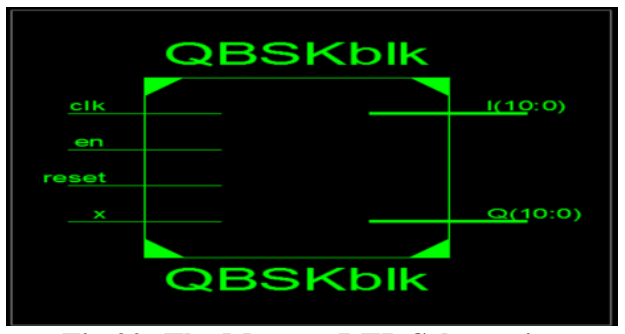

Fig 20: The Mapper RTL Schematic.

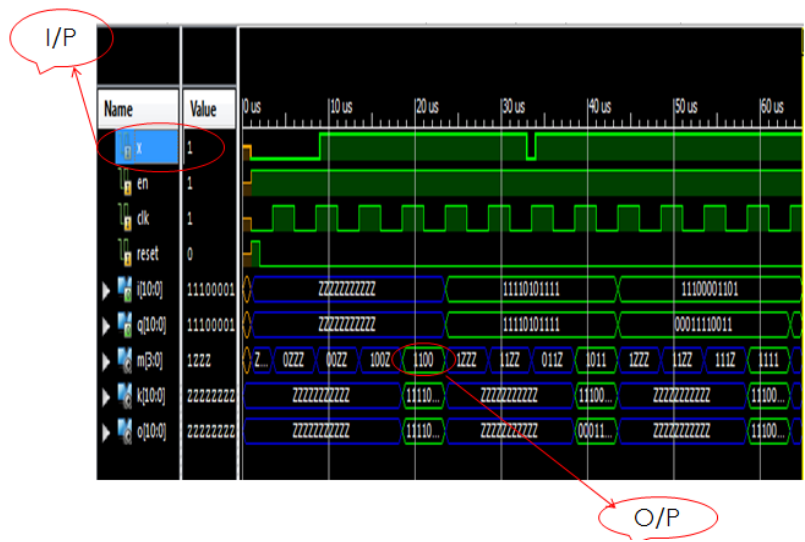

Fig 21: Testing results of the QAM mapper

\subsubsection{Data and Pilot Insertion sub block}

The Data \& Pilot Insertion block is mainly a RAM of length 256 words and width 22 bits. According to the specifications, we use total 180 locations, with 150 for mapped data plus 30 pilots, in the middle of the RAM and the remainder locations will be filled with zeros. The pilots and data are arranged such that every pilot address is followed by five data addresses. The testing results of the implemented Data \& Pilot Insertion sub block are shown in Figure 22.

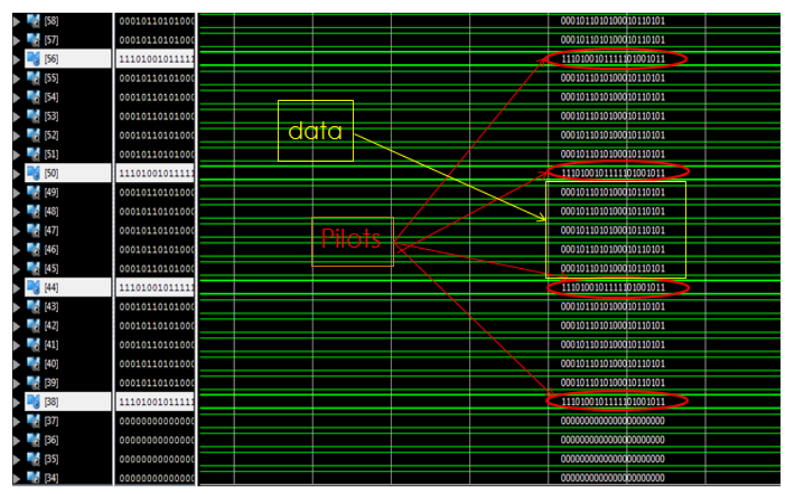

Fig 22: Testing results of the data and pilot insertion sub block

\subsubsection{IFFT and Cyclic Prefix sub block}

The last two operations in the transmitter increase the bandwidth efficiency through orthogonality by using the Inverse Fast Fourier Transform IFFT and preventing the transmitted symbols from ISI and ICI through the insertion of Cyclic Prefix CP. Both the IFFT block and the CP block with the desired specifications are automatically generated by Xilinx core. For the implementation, 256 IFFT size $(38 * 2$ NULLS + 180 data and pilots) was used. Then the output is 256 samples for the IFFT. Figure 23a and 23b show the test signals the implementation using IFFT Xilinx core. While Figure 24 shows the implementation of the $\mathrm{CP}$ by Xilinx core according to the specifications. For short $\mathrm{CP}$, one repeats the last 144 samples at the start of ODFM symbol. 


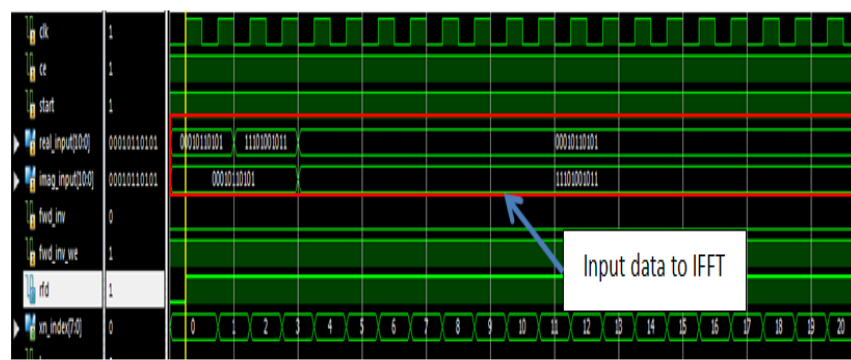

Fig 23a: The input to the IFFT block

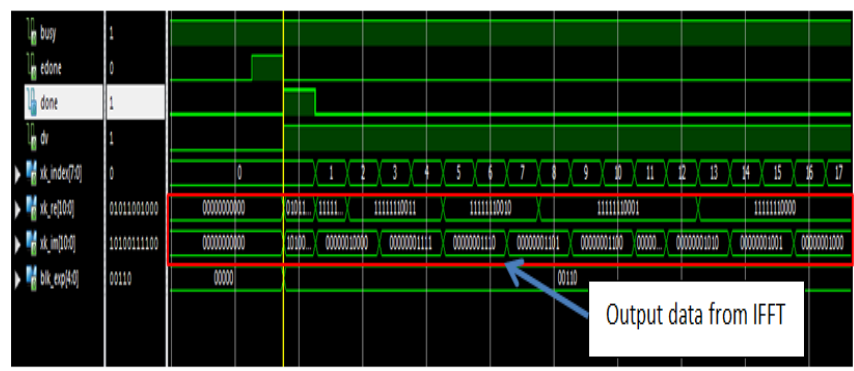

Fig 23b: The output from the IFFT block

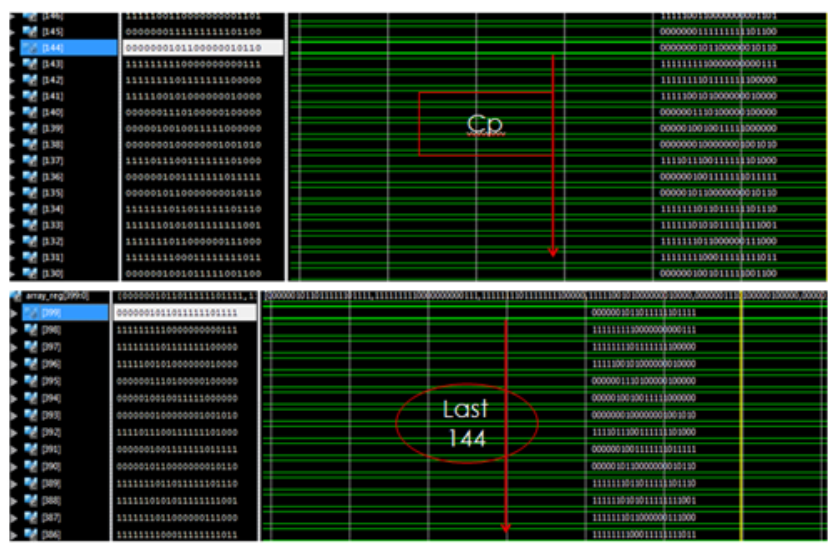

Fig 24: The Cyclic Prefix process

\subsection{THE DE-OFDM BLOCK}

The DE-OFDM block performs the reverse operations of the OFDM block which are cyclic prefix removal, FFT, and DEmapping. The VHDL implementation of these sub blocks will be outlined in the next subsection.

\subsubsection{Cyclic prefix remove and FFT}

The Cyclic prefix removal sub block removes the first repeated 144 samples of the received data. The FFT of the remaining OFDM symbol is implemented using a Xilinx core. Figure 25 shows the IFFT and FFT functionality verification by the loop back process. The IFFT and the FFT blocks are verified when the output sequence of the FFT is identical to that at the input of the IFFT. It is clear from the figure that they are the same.
- Data in ifft $=0001011010100010110101$

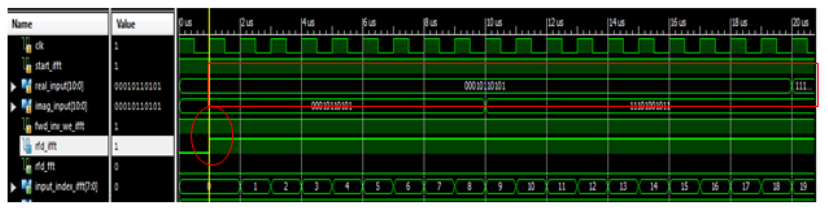

Data out fft=0001001100000010010101

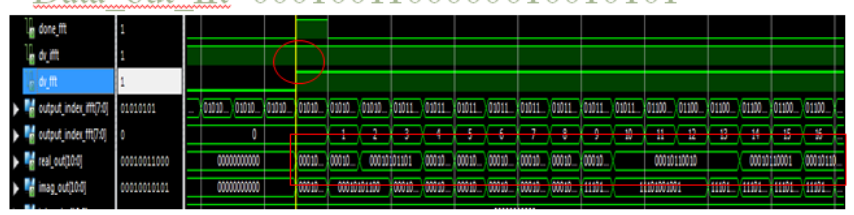

Fig 25: The IFFT and FFT verification

\subsubsection{DE-mapper}

De-mapper at receiver has reverse functionality of the mapper at transmitter, so it receives the complex QAM symbols from the FFT sub block and translates them into logic values in bits. Here, for 4- QAM every complex symbol represented by I and Q values, the output will be 2 bits for every symbol. Figure 26 shows the simulation results of the De-mapper block.

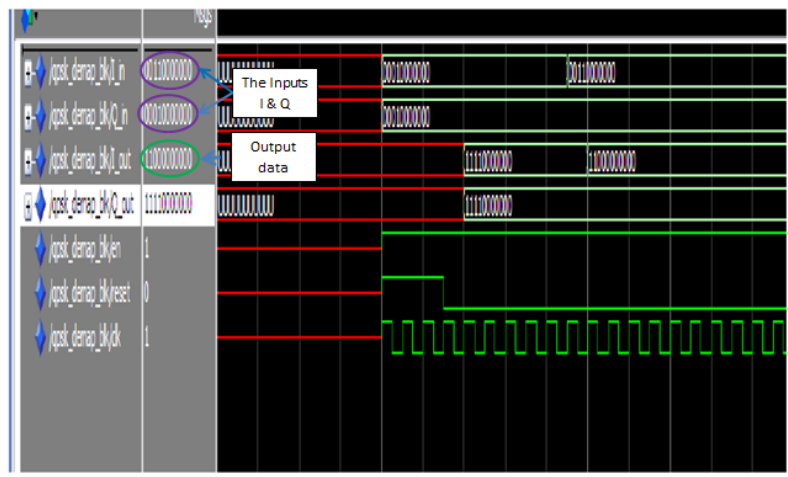

Fig 26: The simulation results of the De-mapper sub block

\subsection{OFDM AND DE-OFDM VERIFICATION}

The OFDM and the DE OFDM verification is passed in the loop back test when the output the DE- OFDM is identical to the input of the OFDM. Figure 27 shows the verification results. It is clear that the output from the De OFDM at the receiver is a delayed version of the input to the OFDM at the transmitter. The delay is due to delay affected by the transmission channel.

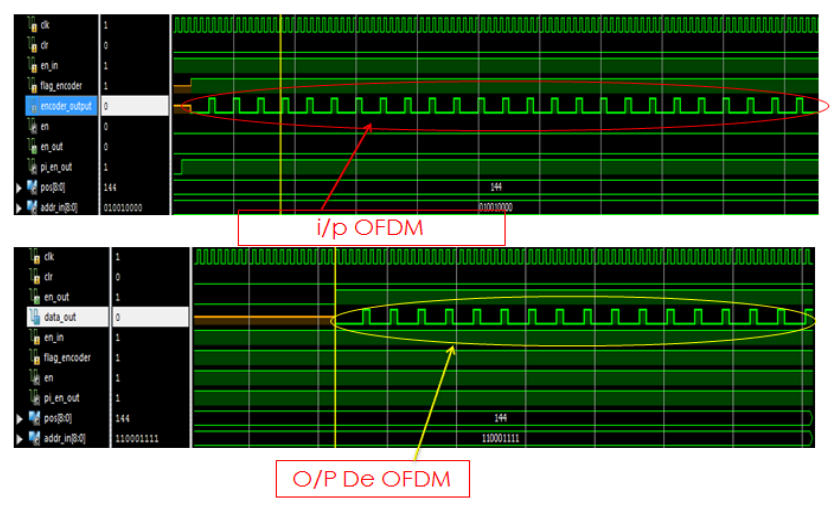

Fig 27: The OFDM and DE OFDM operation outputs. 


\subsection{Time and Frequency Synchronization block}

The function of the Time Synchronization block is to determine the start of the OFDM symbol, while the function of the Frequency Synchronization block is to detect any frequency offset occurred to the carrier due to the channel effect and the mismatch between the receiver oscillator and the received carrier frequency.

The construction of the OFDM symbol is exploited for both Time and Frequency synchronization. We notice that the CP added at the front of any OFDM symbol is a copy from its CP tail an illustrated in Figure 28. To determine the start of the OFDM symbol, we correlated the coming signal with its delayed copy by the length of the OFDM symbol. The length of the correlate is one complete CP of 144 samples. To search for the start of the CP we shift the received symbol by one bit and repeat the correlation process till we obtain a peak. Determining the phase of this maximum one gets the frequency offset of the carrier. Figure 29 illustrates the above described processes carried out for Frequency and Timing synchronization are done through the cross correlation process where the received OFDM symbols are delayed by the OFDM symbol time $\mathrm{T}$, conjugated and complex multiplied by the directly received signal. The output is integrated on the length of the cyclic prefix T0. The point of interest is that where a maximum output is reached. From evaluating the phase of the maximum, one determine the frequency shift of the carrier.

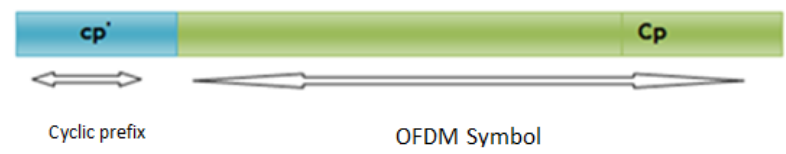

Fig 28: OFDM symbol

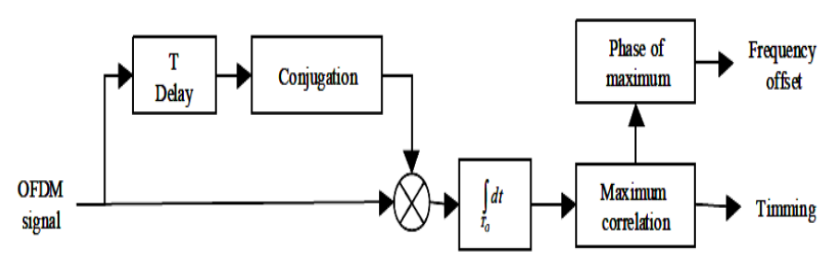

Fig 29: Frequency and Timing Synchronization through cross correlation

VHDL codes for all of the processes in figure 29 are written and the output of the frequency synchronization operation is shown in Figure 30. By inspecting the figure we see that the frequency offset is zero because the channel is modeled only by a time delay only.

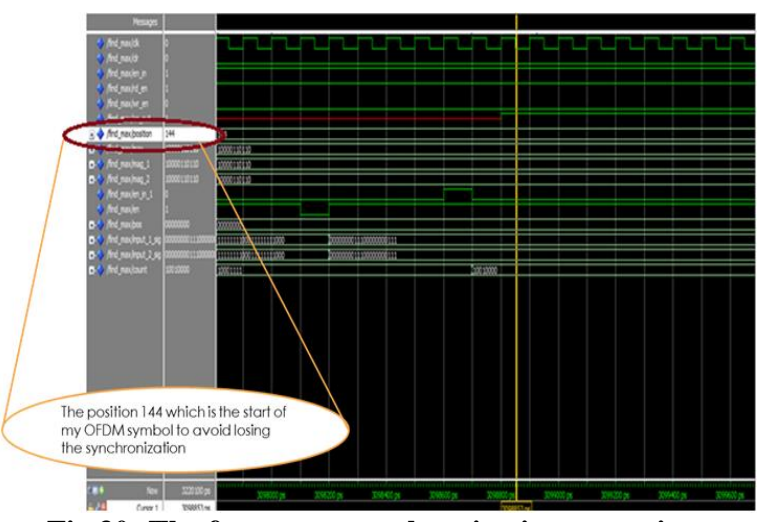

Fig 30: The frequency synchronization operation

The result of the time synchronization operation is shown in Figure 31. One notices that the start of the OFDM symbol is detected at sample number 144 as it should be.

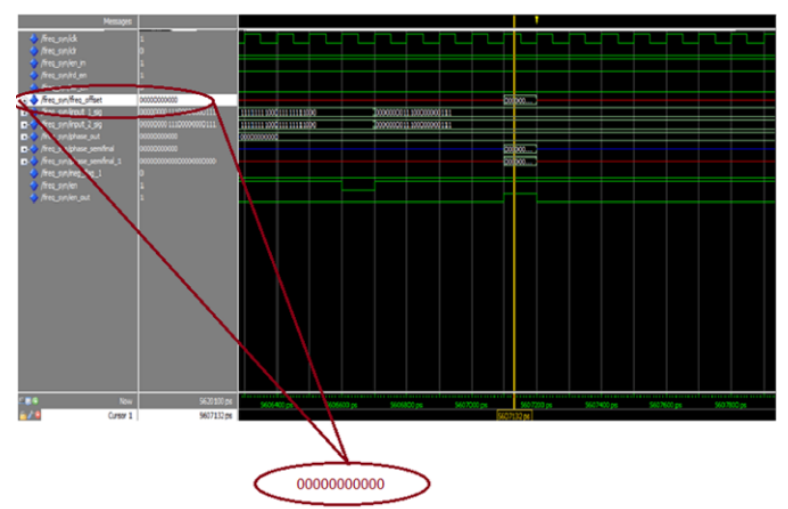

Fig 31: The time synchronization operation

\subsection{CHANNEL ESTIMATION}

The Channel Estimation and equalization block is used to calculate the frequency and phase response of the channel to correct for the effects of the channel impairments due to Path Loss, Multi-path Propagation Fading, and Doppler Effect on the transmitted information in order to recover the transmitted data correctly.

The main building sub blocks of the Channel Estimation are shown in Figure 32. It consists of the sub blocks; Estimations of pilots sub channels, Frequency and time interpolations and Equalization.

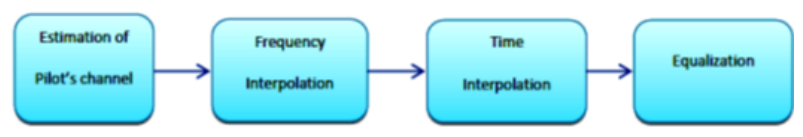

Fig 32: Channel Estimation and equalization general block diagram

The input to the Estimation of Pilots sub Channels block is the Real and Imaginary values of pilots after the channel and the outputs are the Amplitude and phase of the channel response at pilot position. These Real and Imaginary values are converted to the polar form using the CORDIC.

We divide the amplitude of the received Pilots by the amplitude of the original pilot, generated in the receiver, and subtract the Phase of the original pilot from the Phase of the received one. 
The simulation results of the implemented channel Estimation block are shown in Figure 33. It I seen from the figure that the change in amplitude is 1.966 and the change in phase is 0.796 .

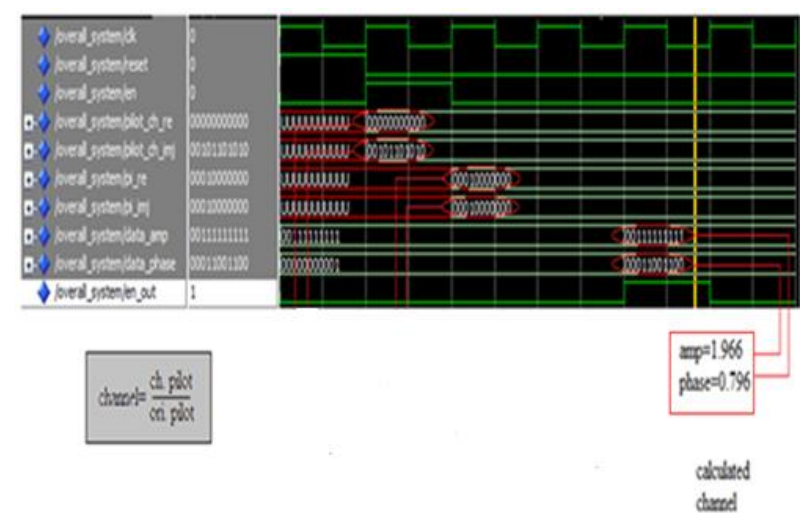

Fig 33: Test results of Estimation of Pilots sub Channels sub block

The function of The Frequency Interpolation sub block is to estimate the channel frequency and phase response on the sub chnnels lying between the pilot sub channels by Linear Interpolation. After interpolating the first OFDM symbol, it receives the values of the next symbol that contain pilots (the fifth OFDM symbol according to the standards) and repeat the same operation .The only difference is that the output of the frequency interpolation block will be stored in different RAM as a preparation step for the Timing Interpolation. The simulation results of the implemented Frequency Interpolation sub block are shown in Figure 34.

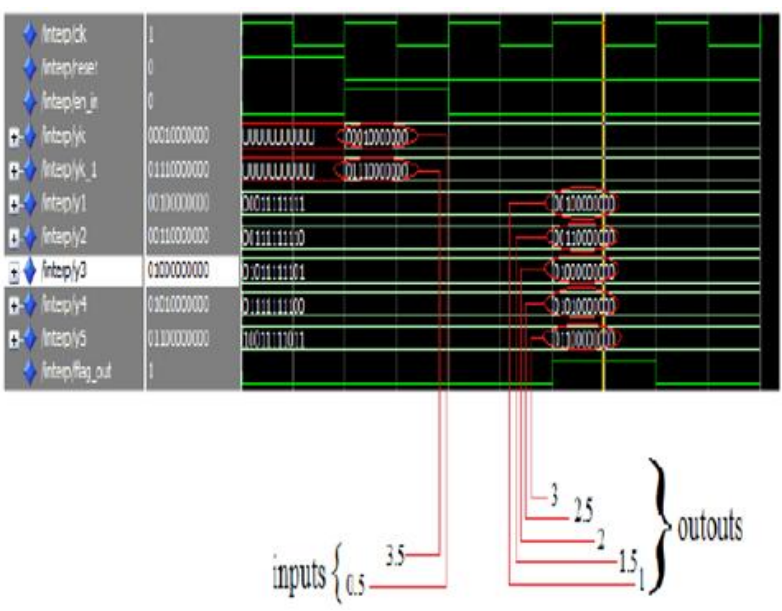

Fig 34: Simulation result of the implemented Frequency interpolation sub block

One sees from the figure the frequency interpolated values between the first and seventh OFDM symbols. The intermediate points assume values according to linear interpolation between 3.5 and 0.5 inputs, respectively.

The Time Interpolation sub block interpolates between values of sub channels in different OFDM symbols to get all other values of channel response in the whole time slot. It estimates the channel response between pilot positions in the same OFDM symbol by Linear Interpolation. The input to the Time Interpolation block is two amplitude values of the same frequency but of different OFDM symbols and the corresponding phase values. The output of the Time Interpolation block is seven interpolated values. The previous steps are repeated $\mathrm{N}$ times such that $\mathrm{N}$ equals the sub channels in one OFDM symbol. All the operations that are made for amplitudes are made for the phase at the same time.

The simulation results of the implemented Time Interpolation sub block are shown in Figure 35.

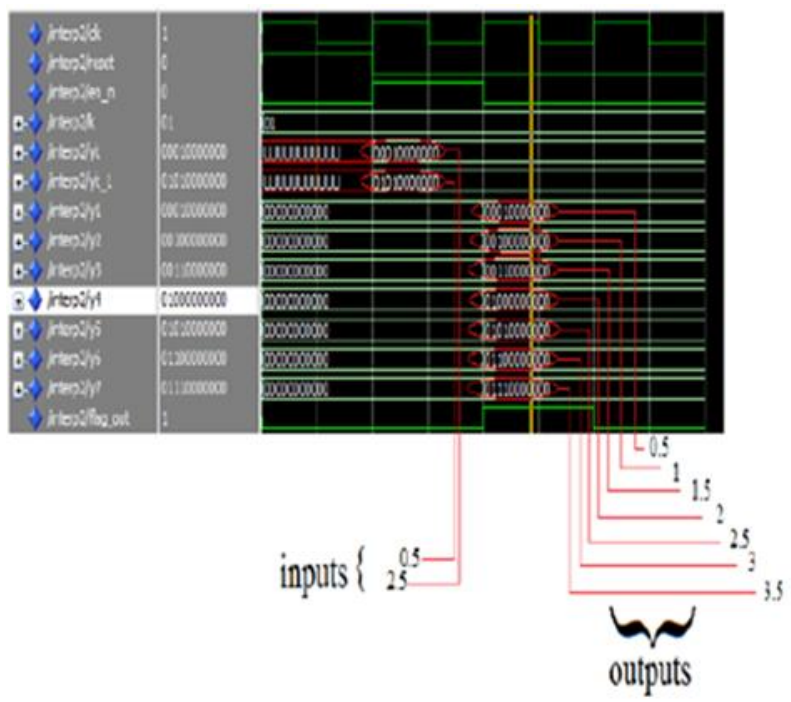

Fig 35: Simulation result of the implemented Time Interpolation sub block

One sees clearly the required linearly interpolated 5 values between 0.5 and 2.5. The same can be done for all sub channels.

Now all the intended values of channel response are available and ready for the next Equalization step.

The Equalization block divides the value of the received data by the channel response to cancel out the channel effect. The division process is done using the CORDIC method. The inputs to this block are the Phase and amplitude of channel response as well as the amplitude and phase of data output from the FFT block. The simulation results of the implemented Equalization sub block are depicted in Figure 36.

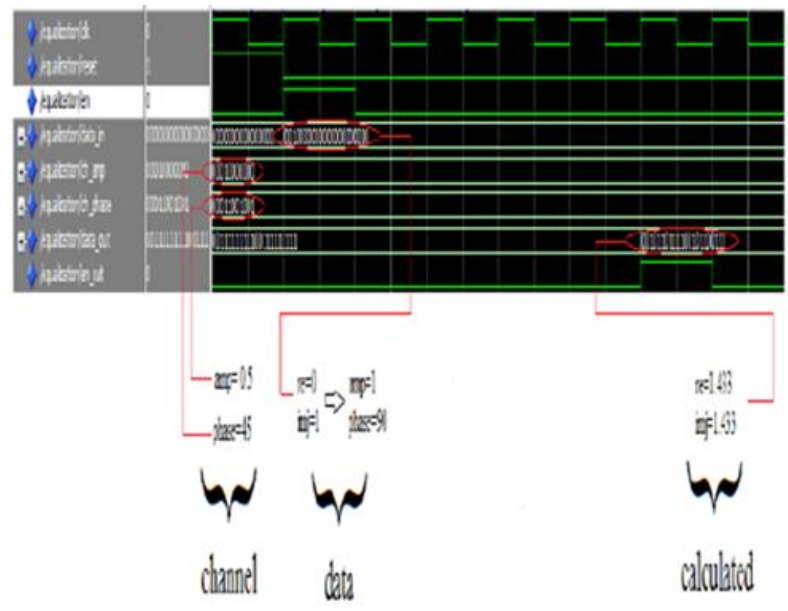

Fig 36: The equalization implementation.

\section{SYSTEM VERIVICATION}

In the previous sections, the correct operations of the implemented stages were ensured on both the block level and 
on the system level. The verification on the block level is done for the main building blocks. If the output of a certain block in the receiver is the same as the input to the corresponding block in the transmitter, then the implementation of these two block pairs is verified. Also, if the final output of the receiver is a delayed version of the input of the transmitter after adding the channel effect; which is represented by a delay of $5 \mu \mathrm{sec}$, then the operation of the overall system is verified.

\section{The overall system verification}

All building blocks of the transmitter are integrated together to build the whole transmitter and the same is carried out for the receiver. The overall system is schematically shown in Figure 37 with a transmission channel having a delay of 5 microseconds. The overall system verification was done by comparing the transmitter input to the receiver output. The input to the transmitter is two OFDM symbols. Figure 38 shows the input data to the Transmitter while Figure 39 shows the output of the Receiver. It is noticed that the data output from the receiver is the same as the data input to transmitter after a delay of $5 \mu$ s of the channel.

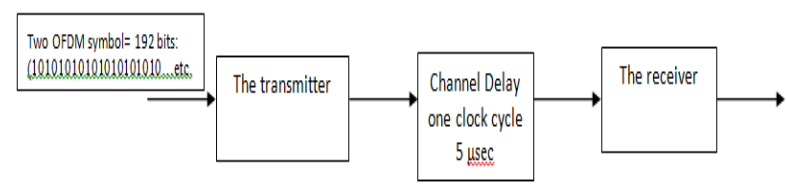

Fig 37: The overall transceiver system block diagram
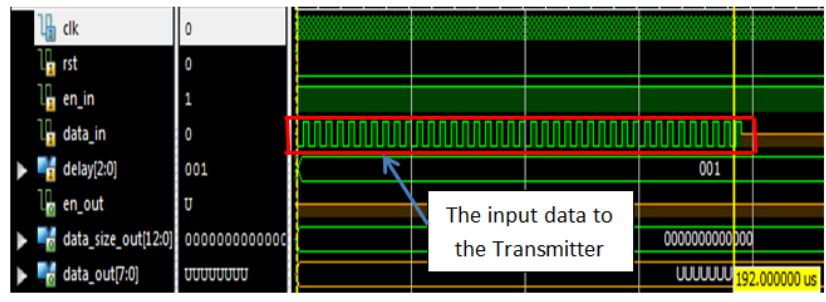

Fig 38: The transmitter input

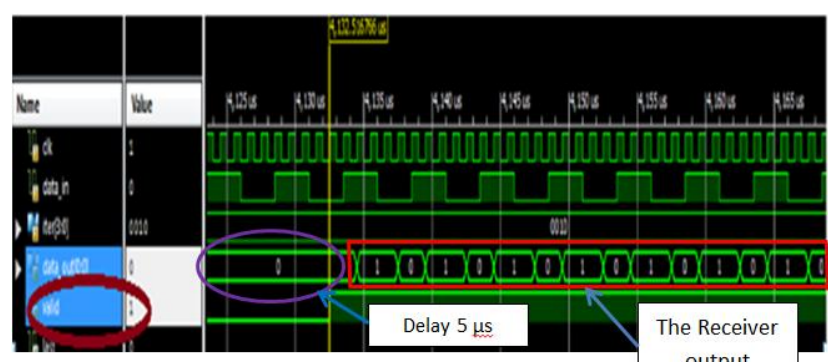

Fig 39: The receiver output

\section{THE SYSTEM UTALIZATION}

After implementing the whole transceiver on Xilinx Virtex 6 , it is interesting to get out the chip utilization for the transmitter and for the whole system.

\subsection{THE TRANSMITTER UTALIZATION}

The Transmitter utilizes the resources given in Table 2 from the Virtex 6 FPGA. It is clear from the table that the LTE transmitter physical layer utilizes a small fraction of resource blocks from Virtex 6.
Table 2. The Transmitter utilization on Virtex 6

\begin{tabular}{|c|c|c|c|c|}
\hline \multicolumn{4}{|c|}{ Device Utitization Sunmary (estimated values) } & E. \\
\hline Logic Utilization & Used & Avalibble & Utitization & \\
\hline Number of flice Regsters & 11447 & 30140 & & $3 \%$ \\
\hline Number of flice UTs & 12021 & 150720 & & $7 \%$ \\
\hline Number of fully used UT.FF pairs & 2860 & 20608 & & $13 \%$ \\
\hline Number of bonded 1008 & 29 & 400 & & $7 \%$ \\
\hline Number of fUJGGGUVGCCTRLs & 3 & 32 & & $9 \%$ \\
\hline
\end{tabular}

\subsection{THE FULL SYSTEM UTALIZATION}

The full system Transmitter and receiver resources utilization table from Virtex 6 FPGA is shown in Table 3. It is clear from the table that the LTE physical layer utilizes a small fraction of resource blocks from Virtex 6 except the lookup tables plus FF pairs where their utilization amount to 62 percent much greater than the other logic blocks. The chip can accommodate more building blocks to complete the control layers of the systems. One may modify the design of the FPGA chips to contain more look up tables more the other logic blocks to equalize the utilization of all blocks and make better utilization of the chip resources. One may redesign system to more equalization of the utilization of the resources on FPGAs. It is anticipated that thi result is useful and very important for the FPGA vendors and logic designers.

Table 3. The Full System Utilization on Virtex 6

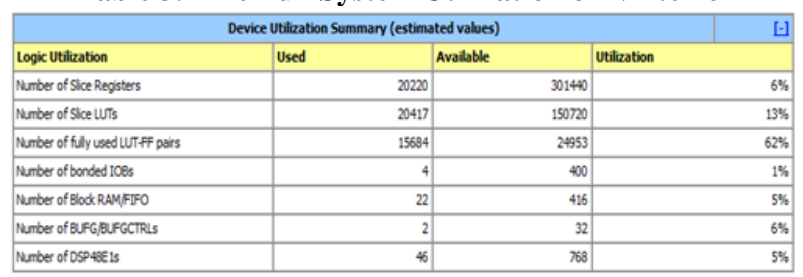

\section{CONCLUSION}

In this paper a complete LTE base band transceiver has been designed as a future mobile technology to cope with next user requirements. All the stages of the LTE Release 9 downlink physical layer including the transmitter and the receiver are modeled using Xilinx® ISE® Design Suite version 12.1 and implemented on Virtex 6 XC6VLX240T FPGA kit. The implemented building blocks include the synchronization and equalization function in the receiver. It is found that the whole LTE downlink physical layer consumes a small fraction of the all logic blocks except the lookup tables and the pair. Their utilization amounts to 62 percent much higher than the other logic resources on the chip. The chip can accommodate more building blocks to complete the control layers of the systems. One may modify the design of the FPGA chips to contain more look up tables more than the other logic blocks to equalize the utilization of all blocks and make better utilization of the chip resources. One may redesign the system for more equalization of the utilization of the resources on FPGAs. It is anticipated that this result is useful and very important for the FPGA vendors and logic designers.

\section{FUTURE WORK}

Future work is aimed to perform the complete LTE physical layer implementation, including the uplink scenario and focusing on the ongoing changes in the LTE specification process; the enhancement can be done with the LTE-advanced starting with Release 10 Specifications. 


\section{REFERENCES}

[1] Jim Zyren,Wes McCoy, "Overview of the 3GPP Long Term Evolution Physical Layer", White Paper, freescale semiconductor.

[2] Y. Tsai. G. Zhang. D. Grieco. F. Ozluturk, "Cell Search in 3GPP Long Term Evolution Systems", IEEE Vehicular Technol. Magazine, vol.2, no.2, June 2007, pp.23-29.

[3] Rohde Schwarz, "UMTS Long Term Evolution (LTE) Technology Introduction", Rohde Schwarz, Application Note 1MA111, Sep. 2008.

[4] Motorola, Inc.," Long Term Evolution (LTE): A Technical Overview", Motorola, Inc., Technical White Paper, 2007.

[5] S. Maruyama, S. Ogawa, K. Chiba, "Mobile Terminals toward LTE and Requirements on Device Technologies", IEEE Symposium on VLSI circuit, pp. 2-5, June 2007.

[6] "LTE: The Future of Mobile Broadband Technology", white paper, Verizon wireless.

[7] LTE; Evolved Universal Terrestrial Radio Access (EUTRA); Physical channels and modulation (3GPP TS 36.211 version 8.3.0 Release 8), ETSI TS 136211 V8.3.0 (2008-11), Technical Specification.

[8] Sung-won Kim, Kun-yong Kim, "Physical layer verification for 3GPP LTE (FDD)", Agilent Technologies.

[9] Christian Mehlf $\square$ uhrer, Martin Wrulich, Josep Colom Ikuno, Dagmar Bosanska, Markus Rupp "SIMULATING THE LONG TERM EVOLUTION PHYSICAL LAYER", 17th European Signal Processing Conference (EUSIPCO 2009).

[10] A.Manikandan,V.Venkataramanan,

M.Kavitha,

\begin{abstract}
S.Parvathi," PERFORMANCE ANALYSIS OF LTE PHYSICAL LAYER BASED ON RELEASE $8 \& 9$ THROUGH SIMULINK ENVIRONMENT", International Journal of Advanced Technology \& Engineering Research (IJATER).
\end{abstract}

[11] 3rd Generation Partnership Project; Technical Specification Group Radio Access Network; Evolved Universal Terrestrial Radio Access (E-UTRA); Physical Channels and Modulation, Release 9, 3GPP TS 36.211 V9.0.0 (2009-12), Technical Specification.

[12] 3rd Generation Partnership Project Technical Specification Group Radio Access Network; Evolved Universal Terrestrial Radio Access (E-UTRA);( Multiplexing and channel coding (Release 9), 3GPP TS 36.212 V9.4.0 (2011-09), Technical Specification.

\section{AUTHOR'S PROFILE}

Sara M. Hassan was born in Cairo, Egypt, on September 8, 1985. She received the B.Sc. degree in Electrical Engineering from Modern academy for engineering and technology, Cairo, Egypt, in 2007, and the M. Sc. degree in Advanced Mobile Communication Techniques from Ain Shams University, Cairo, Egypt, in 2013.She is currently a $\mathrm{PhD}$. student in the Department of Electronics and Communications Engineering at Ain Shams University. Her general research interests include design and implementation for Building Blocks in Advanced Mobile Communication Techniques, and currently her research work focuses on LTE-Advanced transceivers.

Abdelhalim Zekry is a professor of electronics at faculty of Engineering, Ain Shams University, Egypt. He worked as a staff member on several universities. He published more than 160 papers. He also supervised more than 70 Master thesis and 25 Doctorate. Prof. Zekry focuses his research programs on the field of microelectronics and electronic applications including communications and photovoltaics. He got several prizes for his outstanding research and teaching performance. 